\title{
Effects of alcohol on successive incentive contrast
}

\author{
W. MILES COX \\ Veterans Administration Medical Center, Indianapolis, Indiana \\ and Indiana University School of Medicine, Indianapolis, Indiana
}

\begin{abstract}
The effects of alcohol on successive positive- and negative-incentive contrast effects resulting from shifts in magnitude of food reward were studied in a runway conditioning experiment with female albino rats. Immediately prior to the conditioning trials, half of the fluid-deprived animals consumed a $10 \%$ sucrose solution, and half consumed a $10 \%$ sucrose solution containing $4.8 \%$ ethanol. A negative-incentive contrast effect occurred under both fluid conditions, but a positiveincentive contrast effect did not occur under either fluid condition. Thus, there was no evidence that alcohol either eliminated negative emotional reactions to downshifts in reward or contributed to positive emotional reactions to upshifts in reward. On the contrary, consuming alcohol had negative consequences on runway performance, causing the successive negative contrast effect to be more protracted than it was among the animals that did not consume alcohol. The results suggest that consuming alcohol following incentive reduction interferes with animals' disengagement from the lost incentive.
\end{abstract}

Incentive contrast effects resulting from successive shifts in magnitude of food reward have been studied extensively in animals (Black, 1976; Cox, 1975; Flaherty, 1982). With the successive incentive shift design, animals are trained to traverse a runway during two successive phases of the experiment. During Phase 1, experimental animals receive a small (or large) reward until stable, asymptotic running speeds are reached and then during Phase 2 are shifted to a large (or small) reward. The Phase 2 performance of the group shifted from small to large rewards is compared with that of a control group that receives the large reward during both phases (test for positive contrast), and the Phase 2 performance of the group shifted from large to small rewards is compared with that of a control group that receives the small reward during both phases (test for negative contrast). Experimental animals consistently respond more slowly for the small rewards than do control animals that receive only the small rewards (negative-incentive contrast effect). Conversely, experimental animals typically do not respond more rapidly for large rewards than do control animals that receive only large rewards (no positive-incentive contrast effect).

One explanation for incentive contrast effects is that they are mediated by emotional reactions to changes in reward conditions. According to this view, the negative-incentive contrast effect occurs because downshifted animals are depressed (Crespi, 1942) or frustrated (Amsel, 1962) because they do not receive their accustomed large rewards. Similarly, animals receiving an upshift in reward are expected to be elated and exhibit a positive contrast effect (Crespi, 1942). For a discussion of other theoretical explanations of incentive contrast effects as well as explanations for the failure to obtain positive-incentive contrast effects, see Black (1976) and Cox (1975).

This research was supported by a Grant in Aid of Research from the Graduate School of the University of Minnesota. The author wishes to thank Alan Sauter, Phillip Godding, and Kathryn Kurz for their assistance in running the experiments. Requests for reprints should be sent to W. Miles Cox, Psychology Service (116B), Veterans Administration Medical Center, 1481 West 10th St., Indianapolis, IN 46202.
In the present experiment, we tested the effect of alcohol on successive positive- and negative-incentive contrast effects. Since alcohol is a psychoactive drug that modifies emotional reactions, it would be of considerable interest to emotional explanations of contrast effects to determine how alcohol affects incentive contrast effects. From the viewpoint that alcohol reduces negative emotional states (West \& Sutker, in press), alcohol would be expected to counteract the negative emotional reaction to downshifts in reward and thus reduce or eliminate entirely the negative-incentive contrast effect. From the viewpoint that alcohol enhances positive emotional states (Cox, 1981), alcohol would be expected to contribute to rats' elation following upshifts in reward and thereby foster the development of a positive-incentive contrast effect. ${ }^{1}$

\section{METHOD}

\section{Subjects}

The subjects were 79 female albino rats (Holtzman Co., Madison, WI) that were approximately 90 days old at the beginning of training.

\section{Apparatus}

The apparatus was a single straight runway, previously described by Cox and Martz (1985).

\section{Design}

Eight groups were run. ${ }^{2}$ Throughout the experiment, four groups drank an alcoholic fluid prior to their conditioning trials and four groups drank a nonalcoholic fluid. Within each fluid condition, two experimental and two control groups were run. One experimental group received a small food reward during Phase 1 and a large food reward during Phase 2 . The second experimental group received a large reward during Phase 1 and a small reward during Phase 2 . One control group received a small reward during both phases, and the other received a large reward during both phases. These eight groups provided a test for successive positive contrast and successive negative contrast when animals were and were not under the influence of alcohol. The groups are designated in terms of the fluid consumed prior to the runway trials and the magnitude of reward received in the runway during each phase (e.g., Group Alcoholic/ Small-Large drank the alcoholic fluid and received the small reward during Phase 1 and the large reward during Phase 2). 


\section{Procedure}

Upon receipt from the supplier, the animals were housed in individual home cages and were given ad-lib food and water until their weights stabilized. Then the animals were placed on a food-deprivation regimen, consisting of $9 \mathrm{~g}$ of food each day, until their body weights were reduced to $85 \%$ of their ad-lib values. Compensatory food was given whenever the weights fell below $85 \%$. The animals continued to have ad-lib water until 5 days prior to experimental training, when a fluiddeprivation regimen was instituted. On Day 1 of fluid deprivation, all animals were exposed to tap water for $15 \mathrm{~min}$. Throughout the remainder of the experiment, the animals were exposed to either an alcoholic or a nonalcoholic fluid for $15 \mathrm{~min}$ daily.

The purpose of fluid deprivation was to facilitate the animals' consumption of the $4.8 \%$ alcoholic fluid that they received prior to experimental testing. The alcoholic and nonalcoholic fluids were the same as those used by Cox (1981).

On the day prior to the beginning of training, the animals explored the runway for $5 \mathrm{~min}$ in groups of 5 animals each. During training, the animals were tested in squads of 9 or 10 animals each. Each animal had the alcoholic or nonalcoholic fluid in its home cage for $15 \mathrm{~min}$ immediately prior to the conditioning trials in the runway. The fluids, always at room temperature, were presented in graduated cylinders that contained ball-bearing drinking spouts to minimize leakage and thus facilitate accurate measurements of the amount of fluid consumed. When the 15-min exposure duration had elapsed, the squad of animals was wheeled into a dimly illuminated experimental room. Each animal was placed into the runway facing away from the startbox door, and as soon as it had oriented toward the door, the door was opened to permit traversal of the alley. When the animal had entered the goalbox, the goalbox door was closed to prevent reentry into the alley. Small and large food rewards consisted, respectively, of 1 and $1045-\mathrm{mg}$ Noyes food pellets. When it had completely consumed the reward, each animal was removed from the goalbox and returned to the carrying cage to await the next trial. Each animal received four daily trials with an intertrial interval of about $15 \mathrm{~min}$. When each squad's daily trials were completed, the animals were weighed and returned to their home cages and provided with compensatory food when their body weights had fallen below the $85 \%$ ad-lib values. Phases 1 and 2 lasted for 12 days each. At the beginning of Phase 2, the four experimental groups received the appropriate shift in magnitude of food reward. Immediately following Phase 2, all groups were given four daily extinction trials for 6 days. The animals continued to drink the fluid that they had consumed during Phases 1 and 2 , but they received no food in the goalbox.

\section{RESULTS}

The animals showed gradual increases in body weight during Phase $1[F(11,781)=20.64, p<.001]$ and Phase 2 $[F(11,781)=1.96, p<.03]$, but there were no group differences in body weights during either phase $(F<1.0$, in both cases). The animals weighed an average of $198.7 \mathrm{~g}$ during Phase 1 and $208.8 \mathrm{~g}$ during Phase 2.

During the course of both phases of the experiment, there were gradual increases in the volume of fluid consumed $[F(11,781)=33.18, p<.001$, for Phase 1 , and $F(11,781)$ $=10.33, p<.001$, for Phase 2]. Moreover, during both phases the groups that drank the nonalcoholic fluid consumed more fluid than the groups that drank the alcoholic fluid. Mean daily consumption of the two fluids during Phase 1 was $21.8 \mathrm{ml}$ and $15.5 \mathrm{ml}$, respectively $[F(1,77)=88.92$, $p<.001$, and during Phase $2,26.2 \mathrm{ml}$ and $20.5 \mathrm{ml}$, respectively $[F(1,77)=52.84, p<.001]$. Besides the difference in amount of alcoholic and nonalcoholic fluids consumed, there were no meaningful group differences in fluid consumption. Moreover, changes in fluid consumption did not parallel the shifts in rewards during Phase 2. Comparison of each upshifted and downshifted group with its respective control group during Phase 2 revealed no significant group differences in amount of fluid consumption.
Prior to analysis, all runway latencies were converted into speeds $(\mathrm{cm} / \mathrm{sec})$. In turn, each animal's four daily trials were averaged, and all analyses were computed from these averages. Results for run and goal speeds are similar, but group differences are more pronounced for goal speeds, and only they are presented here.

Goal speeds during Phase 1 of the four groups consuming the nonalcoholic fluid are shown in Figure 1a. The four groups showed increases in goal speeds during Phase 1, group differences developed as training progressed, and the four groups appear to have reached asymptotic speeds by the end of Phase 1. Accordingly, an ANOVA indicated that there were highly significant effects for days $[F(11,385)=$ $177.05, p<.001]$ and for groups $[F(3,35)=5.29$, $p<.004]$, but no interaction of days and groups $(F<1.0)$.

Goal speeds during Phase 1 of the four groups consuming the alcoholic fluid are shown in Figure 1b. These four groups showed increases in goal speeds across days $[F(11,396)=224.24, p<.001]$ and appear to have stabilized by the end of Phase 1 at speeds comparable to the asymptotic speeds achieved by the groups drinking the nonalcoholic fluid. Differences among the alcoholic groups are apparent $[F(3,36)=3.33, p<.03]$, but reward-magnitude effects are not as strong as we observed among the nonalcoholic groups.

Goal speeds during Phase 2 of the four groups consuming the nonalcoholic fluid are shown in Figure 1c. Pronounced groups, days, and groups $\times$ days effects were all confirmed by ANOVA $[F(3,35)=7.02, p<.001$; $F(11,385)=7.72, p<.001 ;$ and $F(33,385)=2.72$, $p<.001$, respectively]. Comparison of the Phase 2 performance of the group shifted from large rewards during Phase 1 to small rewards during Phase 2 with that of Group Nonalcoholic/Small-Small indicates that a pronounced negative contrast effect occurred. At the beginning of Phase 2, Group Nonalcoholic/Large-Small showed immediate and precipitous reductions in goal speeds that were depressed far below the speeds of Group Nonalcoholic/Small-Small. Thereafter, there was a rapid recovery in the goal speeds of Group Nonalcoholic/Large-Small so that they soon approximated the goal speeds of Group Nonalcoholic/Small-Small. An ANOVA of the speeds of these two groups during the first half of Phase 2 indicated a significant effect for groups $[F(1,17)=4.99, p<.04]$ and a group $X$ days interaction $[F(5,85)=1.89, p<.10]$. However, no such significant effects occurred during the second half of Phase 2. Further inspection of Figure 1c indicates that the group shifted to large reward during Phase 2 from small reward during Phase 1 did not show a positive contrast effect. At the beginning of Phase 2, Group Nonalcoholic/Small-Large showed increases in goal speeds and soon reached the level of performance of the large-reward control group (Group Nonalcoholic/Large-Large) but did not exceed the performance of this group. An ANOVA of the Phase 2 performance of these two groups revealed neither an effect for groups $(F<1.0)$ nor a groups $\times$ days interaction $[F(11,198)=1.07, p<.38]$.

Goal speeds during Phase 2 of the four groups trained under the alcoholic fluid are shown in Figure 1d. Here we see strong effects for groups $[F(3,36)=6.03, p<.002]$, days $[F(11,396)=2.77, p<.002]$, and the groups $\times$ days interaction $[F(33,396)=2.45, p<.001]$. Inspection of the Phase 2 performance of the group that was shifted to small reward during Phase 2 from large reward during Phase 1 indicates clearly that negative contrast occurred. At the be- 

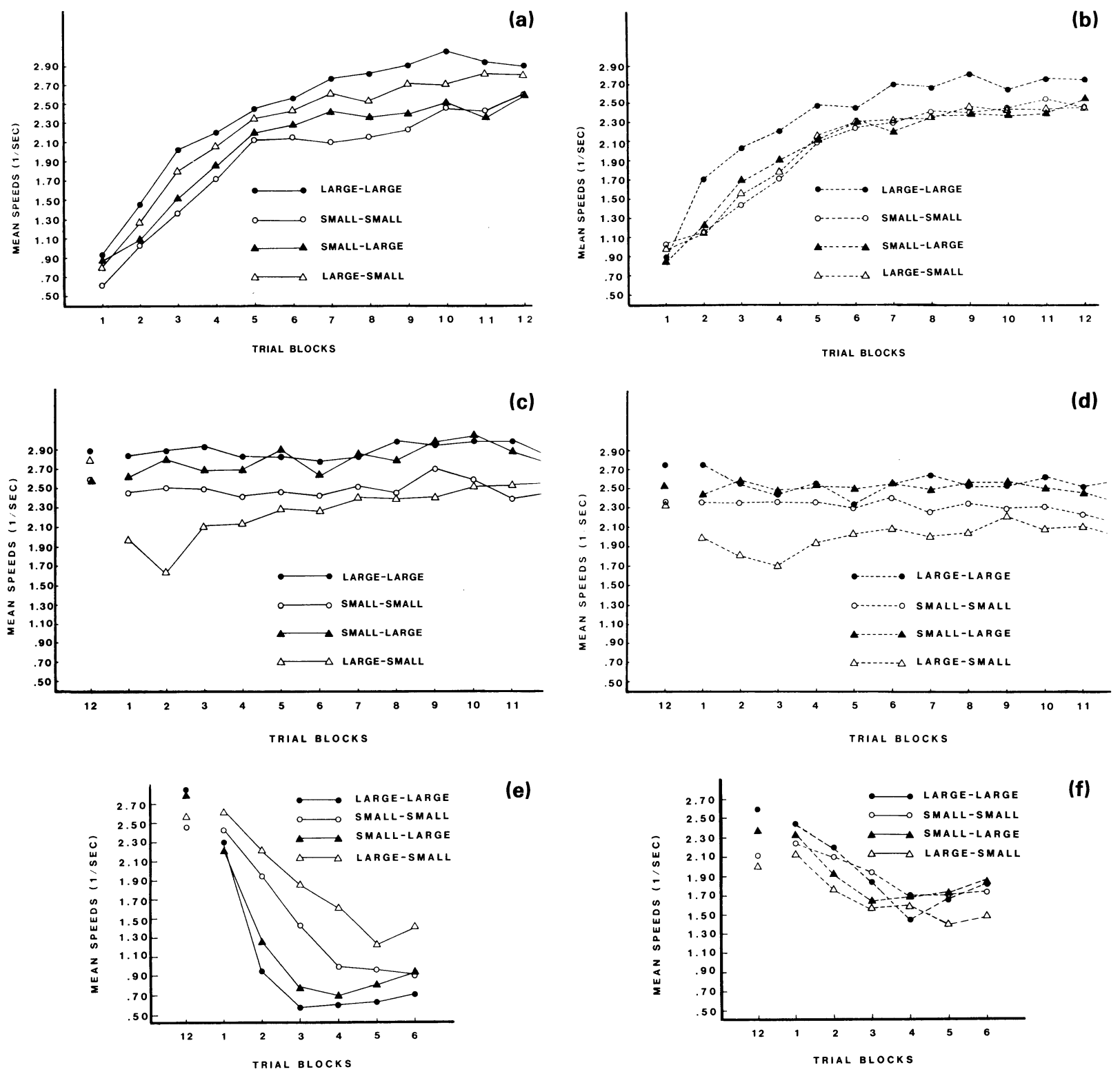

(e)

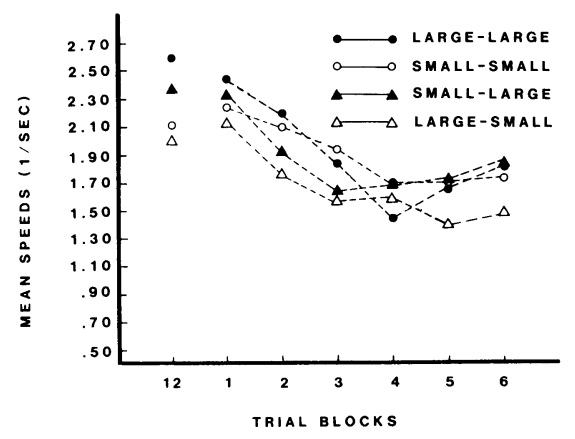

(f)

Figure 1. Goal speeds of the four groups of animals that consumed (a) the nonalcoholic fluid during Phase 1, (b) the alcoholic fluid during Phase 1, (c) the nonalcoholic fluid during Phase 2, (d) the alcoholic fluid during Phase 2, (e) the nonalcoholic fluid during the extinction phase, and (f) the alcoholic fluid during the extinction phase.

ginning of Phase 2 , the goal speeds of Group Alcoholic/Large-Small were depressed below the speeds of Group Alcoholic/Small-Small and continued to decline for several days. Thereafter, recovery was very slow, and, in fact, Group Alcoholic/Large-Small had not reached the level of performance of Group Alcoholic/Small-Small by the end of Phase 2. Statistical comparison of the Phase 2 performance of these two groups confirmed that there was a reliable effect for groups $[F(1,18)=5.60, p<.03]$, days $[F(11,198)$ $=2.51, p<.006]$, and the groups $\times$ days interaction $[F(11,198)=4.28, p<.001]$. On the other hand, inspection of the performance of the group shifted to large reward during Phase 2 after receiving small reward during Phase 1 indicates that no positive contrast occurred. At the beginning of Phase 2, Group Alcoholic/Small-Large showed increases in goal speeds and quickly reached the speeds achieved by Group Alcoholic/Large-Large but did not ex- ceed the performance of this group. In fact, we see that these two groups performed at comparable speeds throughout Phase 2, an observation that is confirmed by statistical analysis $(F<1.0)$.

Goal speeds during the extinction phase of the four groups consuming the nonalcoholic fluid and the four groups consuming the alcoholic fluid are shown in Figures le and 1f, respectively. Here we see that, on the whole, the animals consuming the nonalcoholic fluid extinguished more rapidly than the animals consuming the alcoholic fluid. In addition, the former animals, unlike the latter, showed wide group differences. Accordingly, a factorial ANOVA indicated a significant effect for fluid consumed $[F(1,70)=26.42$, $p<.001]$, as well as an interaction of fluid and reward $[F(3,70)=6.31, p<.001]$. The group differences among the nonalcoholic groups were such that the two groups that had received large reward during Phase 2 extinguished more 
rapidly than the two groups that had received small reward during Phase $2[F(1,37)=18.62, p<.001]$. However, the group that had been upshifted (Nonalcoholic/Small-Large) did not differ from the large-reward control group (Nonalcoholic/Large-Large) during extinction $[F(1,17)=2.83$, $p<.11$, and the group that had been downshifted (Nonalcoholic/Large-Small) did not differ from the small-reward control group (Nonalcoholic/Small-Small) $[F(1,18)=1.37$, $p<.26]$.

\section{DISCUSSION}

In some respects, animals that consumed the alcoholic fluid and animals that consumed the nonalcoholic fluid prior to their runway conditioning trials reacted in a similar manner to changes in reward conditions. Thus, with the shift from large to small reward, animals trained under both fluid conditions showed a significant negative contrast effect. Conversely, with the shift from small to large reward, neither the animals that drank the alcoholic fluid nor those that drank the nonalcoholic fluid showed a positive contrast effect. In short, the present results indicate neither that alcohol eliminated negative contrast nor that it fostered the development of positive contrast.

Despite the generally similar reactions to shifts in rewards by animals that consumed the alcoholic and nonalcoholic fluids, there were some striking differences between animals in the two fluid conditions in their reactions to different reward conditions. The performance of the animals that consumed the nonalcoholic fluid was typical of that of animals in numerous prior studies of successive incentive contrast effects not involving alcohol. Thus, these animals showed strong and consistent effects for magnitude of reward. Between groups receiving different magnitudes of reward, the animals ran more rapidly for large reward than for small reward. Furthermore, immediately upon being shifted from large to small reward, the animals that did not consume alcohol showed precipitous declines in running speeds that were followed by rapid recovery. A robust negative contrast effect immediately upon being shifted from large to small reward, as well as rapid recovery from the disruption, is typical of previous findings. Finally, the fact that there was no evidence for a positive contrast effect is consistent with the results of prior studies of upshifts in reward not involving alcohol.

Compared with the animals that drank the nonalcoholic fluid, the animals that drank the alcoholic fluid were far less responsive to different magnitudes of rewards. In fact, in some cases there were no differences at all in the speeds of animals that received large and small rewards. Another difference between the animals consuming the alcoholic and nonalcoholic fluids concerns the intensity and duration of the successive negative contrast effect. In the case of the former animals, the initial disruption in performance was less abrupt, suggesting that the alcohol did have some temporary assuaging effect on the negative emotional reaction to the downshift in reward, but the effect was far more protracted, suggesting that alcohol interfered with recovery from the disruption.

There were also differences in the extinction performance of animals in the two fluid conditions. For example, the rate of extinction was far greater for the groups consuming the nonalcoholic fluid than for those consuming the alcoholic fluid. The animals that consumed alcohol performed at comparable speeds during the extinction phase, regardless of what their prior experience with reward had been. On the other hand, the animals that consumed the nonalcoholic fluid showed clear-cut group differences, such that the animals that had received the large reward during the prior phase extinguished more rapidly than animals that had received the small reward during the prior phase.

The preceding conclusions are not consistent with the expected effects of alcohol on emotional reactions following shifts in rewards. Thus, the present results do not support the expectation that alcohol will attenuate negative emotional reactions (depression or frustration) that might mediate negative contrast effects. In addition, no support is gained for the view that alcohol contributes to animals' elation following upshifts in reward that might be responsible for positive contrast. Finally, although consuming alcohol did result in slower extinction, this outcome may not be the result of alcohol's counteracting negative emotional reactions to the elimination of reward. In view of the present animals' failure to show reward-magnitude effects when they had drunk alcohol during the earlier phases of the experiment, it seems likely that their slower responding during the extinction phase resulted from their lack of responsiveness to the change in reward conditions rather than from alcohol's alleviating their negative emotions resulting from that change.

Instead of being consistent with theoretical views about the emotional consequences of alcohol consumption, the preceding conclusions imply that alcohol had maladaptive consequences. That is, the animals generally responded less "appropriately" to incentive conditions when they were under the influence of alcohol. Perhaps the most maladaptive consequence of consuming alcohol was the effect that it had on the successive negative-incentive contrast effect. Alcohol did attenuate the magnitude of the successive negative contrast effect initially, but it interfered with recovery from the disruptive effects of downshifts in reward. Klinger's (1977) theory of incentives offers one possibility for accounting for this outcome. According to the theory, when an organism loses an incentive that it has been committed to pursue, it undergoes an orderly sequence of changes, collectively known as the incentive disengagement cycle, that is reflected both in its emotional state and its general level of activity. Depression is one phase of the cycle that is necessary for the organism to become effectively disengaged from a lost incentive. This analysis implies that pharmacological interventions that relieve depression following incentive loss will hamper the organism's disengagement from the lost incentive and thus will be maladaptive. On the basis of this theory, we might infer that in the present results the negative contrast effect was so protracted because the initial depression was eased. Because alcohol does relieve depression, it would seem reasonable that organisms are motivated to consume alcohol voluntarily following incentive loss or reduction but that the long-term consequences of this consumption are undesirable. Consuming alcohol, that is, seems to retard recovery and return to a "normal" level of functioning.

\section{REFERENCES}

AMSEL, A. (1962). Frustrative nonreward in partial reinforcement and discrimination learning: Some recent history and a theoretical extension. Psychological Review, 69, 306-328.

BLACK, R. W. (1976). Reward variables in instrumental conditioning: A theory. In G. H. Bower (Ed.), The psychology of learning and motivation (Vol. 10). New York: Academic Press.

Cox, W. M. (1975). A review of recent incentive contrast studies involving discrete trial procedures. Psychological Record, 25, 373-393.

Cox, W. M. (1981). Simultaneous incentive contrast effects with alcoholic and nonalcoholic beverages as the discriminanda for reward magnitude. Physiological Psychology, 9, 276-280.

Cox, W. M., \& MARTZ, J. E. (1985). A test for successive incentive contrast effects with a highly preferred fluid reward. Bulletin of the Psychonomic Society, 23, 418-420.

CRESPI, L. P. (1942). Quantitative variations of incentive and performance in the white rat. American Journal of Psychology, 55, 467-517.

FLAHERTY, C. F. (1982). Incentive contrast: A review of behavioral changes following shifts in reward. Animal Learning \& Behavior, 10 409-440.

KLINGER, E. (1977). Meaning and void: Inner experience and the incentives in people's lives. Minneapolis: University of Minnesota Press.

RABIN, J. S. (1975). Effects of varying sucrose reinforcers and amobarbital sodium on positive contrast in rats. Animal Learning \& Behavior, 3, 290-294.

WeSt, J. A., \& SUTKER, P. B. (in press). Alcohol consumption, tension reduction, and mood enhancement. In W. M. Cox (Ed.), Why people drink: Parameters of alcohol as a reinforcer. New York: Gardner Press.

\section{NOTES}

1. For a discussion of the effects of psychoactive drugs other than alcohol on incentive contrast effects, see Cox $(1975$, p. 379), Flaherty (1982), and Rabin (1975).

2. Groups 1-7 consisted of 10 animals each, and Group 8 consisted of 9 animals.

(Manuscript received for publication April 13, 1987.) 